\title{
Hereditary angioedema revisited
}

$\mathbf{I}^{1}$ n a previous issue of the Proceedings we wrote an editorial that recounted the seminal description of hereditary angioedema (HAE) by William Osler in 1888 and cited its importance as an instructive model of clinical description, molecular discovery, and new effective treatments. ${ }^{1}$ This rare, autosomal dominant condition that has a congeries of disease manifestations, including a severe risk for life-threatening laryngeal edema and a significant burden of illness, continues to generate cause for continual publications in the Proceedings. Because of the serious nature of the condition and the importance for immediacy of treatment regardless of anatomic site of disease involvement, Baker et al. ${ }^{2}$ examined the time to the beginning of symptom relief after treatment with recombinant human C1 esterase inhibitor by attack location. Analyzing pooled data from two double-blind clinical trials, the authors reported that recombinant human $\mathrm{C} 1$ esterase inhibitor $50 \mathrm{IU} / \mathrm{kg}$ was efficacious in shortening the median time to the beginning of symptom relief of acute HAE attacks, regardless of attack location (abdominal, peripheral, oro-facial-pharyngeal-laryngeal, or urogenital). Response times ranged from 60 to 119 minutes, depending on the location.

For some patients with HAE, in addition to the readily available acute care therapeutic agents, prophylactic therapies may be necessary for long-term management. ${ }^{3,4}$ In this issue, Li et al. ${ }^{5}$ reported their safety evaluation analysis of the use of subcutaneous $\mathrm{C} 1$ esterase inhibitor to prevent HAE attacks in the COMPACT trial, which allowed for treatment doses of $40 \mathrm{IU} / \mathrm{kg}$ and of $60 \mathrm{IU} / \mathrm{kg}$ twice weekly. The authors reported that subcutaneous C1 esterase inhibitor was safe and well tolerated, with no dose-dependent safety concerns.

In transitioning from HAE to asthma, the article by Arnold et $a .^{6}$ serves to remind readers of the importance of cost considerations in asthma management, which is especially relevant given the current emphasis on cost-effective medical care considerations. Asthma exacerbations are now well recognized to be substantial drivers of asthma costs. According to a recent Cochrane meta-analysis, the use of fractional exhaled nitric oxide (FeNO) monitoring is associated with a $40-50 \%$ reduction in the risk of asthma exacerbations. ${ }^{7}$ On this basis, Arnold et al. ${ }^{6}$ explored the potential for

Copyright (C) 2018, OceanSide Publications, Inc., U.S.A. cost savings attributable to the use of FeNO in a realworld data base of Medicare beneficiaries. They reported cost modeling estimates that demonstrated significant cost savings in association with FeNO monitoring of beneficiaries with a history of asthma exacerbations. $^{6}$

For patients for whom Global Initiative for Asthma ${ }^{8}$ step 4 management failed to achieve asthma control, another efficacious strategy to reduce exacerbations is the therapeutic use of biologic agents for add-on maintenance treatment. This issue of the Proceedings includes two articles that highlight the reduction of asthma exacerbations that resulted from treatment with biologic therapies specifically directed at pathophysiologic pathways that underlie asthma. In the first article, which relates to biologic therapy, DuBuske et al. ${ }^{9}$ investigated the effect of benralizumab on the seasonal variability of exacerbations in patients with severe, uncontrolled eosinophilic asthma. Benralizumab is a humanized monoclonal antibody selective for interleukin-5 receptor alpha subunit that induces direct, rapid, and nearly complete depletion of eosinophils via enhanced antibody-dependent cell-mediated cytotoxicity. The authors performed a post hoc analysis of pooled data from the phase III SIROCCO and CALIMA trials. ${ }^{9}$ In their analysis, they reported that benralizumab significantly and consistently reduced asthma exacerbations across all seasons versus placebo for patients with severe, uncontrolled eosinophilic asthma. ${ }^{9}$ Because of the importance of this article and its clinically useful implications, it was chosen for this issue's "For the Patient" section. This segment, found in the final pages of the print version of this issue and also available online, consists of a one-page article synopsis written in a readily comprehensible fashion to help patients better understand the content of the full article.

In the second biologic therapy article, Liu et al. ${ }^{10}$ performed a meta-analysis of randomized controlled trials that evaluated the efficacy and safety of lebrikizumab in patients with uncontrolled asthma. Lebrikizumab is a humanized, monoclonal antibody that blocks interleukin 13, a cytokine that is thought to induce the expression of periostin, another signaling cytokine produced by bronchial epithelial cells. Periostin, in turn, contributes to airway inflammation, bronchial hyperresponsiveness, and activation and proliferation of airway fibroblasts. The authors reported that, in patients with uncontrolled asthma, lebrikizumab treatment not only significantly decreased the rate of exacerbation but also im- 
proved lung function, especially for patients with high periostin levels. ${ }^{10}$

Inhaler technology has been continuously advancing since the introduction of the first metered-dose inhaler (MDI) in the 1950s. Co-suspension delivery technology is an innovative MDI formulation platform that uses strong, nonspecific associations between drug crystals and microsized, spray-dried, phospholipid porous particles to provide uniform dose delivery of inhaled drugs or drug combinations. With a goal of advancing the use of co-suspension delivery technology in asthma, Miller et al. ${ }^{11}$ conducted a randomized doseranging study of a novel budesonide MDI. When comparing four different budesonide doses (320, 160, 80, and $40 \mu \mathrm{g}$ ) with placebo, the authors reported finding that higher doses of budesonide exhibited greater efficacy, with adverse event profiles similar to those seen at lower doses.

Although increased numbers of mast cells are found in lesional skin of patients with atopic dermatitis, the role of serum basal tryptase (sBT) levels as an important biomarker of atopic dermatitis is unknown. Sahiner et al. ${ }^{12}$ studied the association of sBT levels in patients with atopic dermatitis and disease severity. The results of their multivariate logistic regression analysis indicated that sBT levels of $\geq 3.9 \mathrm{ng} / \mathrm{mL}$ were independently associated with an increased risk of moderate-severe atopic dermatitis. ${ }^{12}$

We shift our attention to the subject of food allergy with two articles: the first is directed at diagnostic testing in shrimp allergy, and the second is focused on patients allergic to camel milk. Tuano et al. ${ }^{13}$ sought to identify component-resolved diagnostic tests useful for the diagnosis of shrimp allergy in patients sensitized to house-dust mite and those not sensitized to house-dust mite. The authors reported that the presence of dust mite sensitivity influenced shrimp specific immunoglobulin E levels, which thereby affected diagnostic accuracy in shrimp allergy. ${ }^{13}$ The second article studied camel milk. Through the effectiveness of Internet marketing, powdered camel milk is now widely available and touted for its nutritional value. Because camel milk possesses distinct structural milk proteins, it has limited cross-reactivity to cow's milk ${ }^{14}$ Ehlayel and Bener ${ }^{15}$ presented nine cases of camel milk allergy, which served to characterize this distinct, though very rare, food allergy.

In transitioning from food allergy to the treatment of anaphylaxis, Moss et al. ${ }^{16}$ presented a prospective study of human factors that have the potential to affect the correct injection technique for administration of a newly approved prefilled syringe of epinephrine (SYMJEPI). Their results indicated that SYMJEPI has features that may provide an additional option among user-friendly treatments for acute anaphylaxis.
This issue's "Patient-Oriented Problem Solving" (POPS) case presentation explores the differential diagnosis of a 45-year-old man with elevated levels of immunoglobulin A. The POPS case presentation is a recurring feature of the Proceedings that, as per tradition, is written by an allergy/immunology fellow-intraining from one of the U.S. allergy/immunology training programs. The purpose of the POPS series is to provide an innovative and practical learning experience for the allergist/immunologist in-training by using a didactic format of clinical presentation and deductive reasoning. In this issue's POPS, Liang et al. ${ }^{17}$ from the allergy-clinical immunology department at the Southern California Permanente Medical Group (Los Angeles) led the reader through this learning process and illustrated the complexity of the differential diagnostic process for this clinical presentation and the importance of a detailed history, physical examination, and appropriate laboratory assessment in arriving at a correct diagnosis.

In summary, the collection of articles found within the pages of this issue provides further insight into the intersecting crossroads of genetics and the environment that manifest as the allergic, cutaneous, and respiratory disorders that afflict patients whom the allergist/immunologist serves. In particular, they exemplify how the complexities of asthma, atopic dermatitis, food allergy, anaphylaxis, HAE, and gammopathies continue to challenge the allergist/immunologist. In keeping with the overall mission of the Proceedings, which is to distribute timely information regarding advancements in the knowledge and practice of allergy, asthma, and immunology to clinicians entrusted with the care of patients, it is our hope that the articles found within this issue will help foster enhanced patient management and outcomes. On behalf of the Editorial Board, we hope that you are able to make practical use of the diversity of literature offered in this issue of the Proceedings.

Joseph A. Bellanti, M.D. and
Russell A. Settipane, M.D.

\section{REFERENCES}

1. Bellanti JA, Settipane RA. Hereditary angioedema: an instructive model of clinical description, molecular discovery, and development of new effective treatments. Allergy Asthma Proc. 2011; 32(Suppl 1):S1.

2. Baker JW, Bernstein JA, Harper JR, Relan A, Riedl MA. Efficacy of recombinant human $\mathrm{C} 1$ esterase inhibitor across anatomic locations in acute hereditary angioedema attacks. Allergy Asthma Proc. 2018; 39:359-364.

3. Weller K, Maurer M, Fridman M, Supina D, Schranz J, Magerl M. Health-related quality of life with hereditary angioedema following prophylaxis with subcutaneous C1-inhibitor with recombinant hyaluronidase. Allergy Asthma Proc. 2017; 38:143151.

4. Gower RG. HAE update: determining optimal patient specific therapy. Allergy Asthma Proc. 2013; 34:7-12. 
5. Li HH, Mycroft S, Christiansen S, et al. Subcutaneous C1esterase inhibitor to prevent hereditary angioedema attacks: safety findings from the COMPACT trial. Allergy Asthma Proc. 2018; 39:365-370.

6. Arnold RJ, Layton A, Massanari M. Cost impact of monitoring exhaled nitric oxide in asthma management. Allergy Asthma Proc. 2018; 39:338-344.

7. Petsky HL, Kew KM, Turner C, Chang AB. Exhaled nitric oxide levels to guide treatment for adults with asthma. Cochrane Database Syst Rev. 2016; 9:CD011440.

8. GINA-Global Strategy for Asthma Management and Prevention, Global Initiative for Asthma (GINA). 2018. Available from www.ginasthma.org.

9. DuBuske L, Newbold P, Wu Y, Trudo F. Seasonal variability of exacerbations of severe, uncontrolled eosinophilic asthma and clinical benefits of benralizumab. Allergy Asthma Proc. 2018; 39:345-349.

10. Liu $\mathrm{Y}$, Zhang S, Chen R, et al. Meta-analysis of randomized controlled trials for the efficacy and safety of anti-interleukin-13 therapy with lebrikizumab in patients with uncontrolled asthma. Allergy Asthma Proc. 2018; 39:332-337.
11. Miller SD, Nyberg J, Siddiqui S, Dorinsky P, Rose ES, Reisner C. Randomized dose-ranging study of a budesonide metered-dose inhaler by using co-suspension delivery technology in asthma. Allergy Asthma Proc. 2018; 39:350-358.

12. Sahiner M, Buyuktiryaki B, Gungor HE, et al. Factors that predict disease severity in atopic dermatitis: the role of serum basal tryptase. Allergy Asthma Proc. 2018; 39:371-376.

13. Tuano KTS, Anvari S, Hanson IC, et al. Improved diagnostic clarity in shrimp allergic non-dustmite sensitized patients. Allergy Asthma Proc. 2018; 39:377-383.

14. Ehlayel MS, Hazeima KA, Al-Mesaifri F, Bener A. Camel milk: an alternative for cow's milk allergy in children. Allergy Asthma Proc. 2011; 32:255-258.

15. Ehlayel M, Bener A. Camel's milk allergy. Allergy Asthma Proc. 2018; 39:384-388.

16. Moss RB, Moll T, Daniels K, Carlo DJ. Human factors study of a newly approved prefilled syringe of epinephrine for the treatment of anaphylaxis. Allergy Asthma Proc. 2018; 39:389393.

17. Liang EH, Lim K, Samant SA, Sheikh J. A 45-year-old man with elevated levels of immunoglobulin A. Allergy Asthma Proc. 2018; 39:394-397. 